\title{
Qualidade de saúde bucal e pneumonia associada à ventilação mecânica
}

\author{
Quality of oral health and pneumonia associated with mechanical ventilation \\ La calidad de la salud oral y la neumonía asociada a la ventilación mecánica \\ Aline Bergman de Souza HERCULANO1 \\ Diego Silva de CASTRO ${ }^{1}$ \\ Ellen Cristina GAETTI-JARDIM2 \\ Júlio César Leite da SILVA ${ }^{3}$
}

${ }^{1}$ Cirurgião-Dentista Residência Multiprofissional em Saúde Atenção ao Paciente Critico Adulto, Hospital Universitário Maria Aparecida Pedrossian - HUMAP/EBSERH-UFMS, 79080-190 Campo Grande-MS, Brasil

${ }^{2}$ Profa.Dra. da Disciplina de Cirurgia Bucomaxilofacial da Faculdade de Odontologia FAODO/UFMS, 79070-900 Campo Grande-MS, Brasil

${ }^{3}$ Prof.Dr. da Disciplina de Cirurgia Bucomaxilofacial da Faculdade de Odontologia FAODO/UFMS, 79070-900 Campo Grande-MS, Brasil

\begin{abstract}
Resumo
A cavidade bucal é a incubadora ideal para o desenvolvimento de microbiota devido ao pH, presença de nutrientes e fatores de retenção que facilitam a maturação do biofilme dentário, microbiota essa que frequentemente encontra-se em harmonia e equilíbrio com o hospedeiro saudável, diferentemente de pacientes hospitalizados sob terapia intensiva. O propósito desse estudo foi avaliar a qualidade da saúde bucal de pacientes internados na unidade de terapia intensiva e comparar com a incidência de pneumonia associada a ventilação mecânica. Foram avaliados todos os prontuários de admissão no Centro de Terapia Intensiva Adulto do Hospital Universitário Maria Aparecida Pedrossian HUMAP/EBSERH, no período de 18 de Agosto a 15 de Novembro de 2016, comparando os devidos dados com os Boletins Mensais de Indicadores emitidos pelo Serviço de Controle de Infecção Hospitalar, que mostra os índices de infecções por pneumonia associada a ventilação mecânica correspondentes ao mesmo período. Foram observados que dos 41 pacientes internados no referido setor no período analisado, 37 (90\%) pacientes foram avaliados pela equipe de odontologia hospitalar, dentre esses $68 \%$ eram do sexo masculino e $32 \%$ eram do sexo feminino, com faixa etária de 30 a 70 anos (62\%), em suporte ventilatório com intubação orotraqueal (61\%), com doenças respiratórias como principal motivo de internação (46\%). A comparação realizada entre a qualidade da saúde oral e a incidência de pneumonia associada a ventilação mecânica não apresentou dados significativos que apontem para uma possível relação entre si, provavelmente devido a atuação da equipe de odontologia hospitalar.
\end{abstract}

Descritores: Saúde Bucal; Pneumonia Associada à Ventilação Mecânica; Unidade de Terapia Intensiva.

\begin{abstract}
The oral cavity is the ideal incubator for the development of microbiota due to $\mathrm{pH}$, presence of nutrients and retention factors that facilitate the maturation of the dental biofilm, a microbiota that is often in harmony and balance with the healthy host, differently of hospitalized patients under intensive care. The purpose of this study was to evaluate the quality of oral health of patients admitted to the intensive care unit and to compare it with the incidence of pne umonia associated with mechanical ventilation. All admission dental records implanted at the Adult Intensive Care Center of the Hospital Universitário Maria Aparecida Pedrossian HUMAP / EBSERH were evaluated from August 18 to November 15, 2016, comparing the data with the Monthly Indicator Bulletins Issued by the Hospital Infection Control Service, which shows the rates of pneumonia associated with mechanical ventilation for the same period. In the present study, 37 (90\%) of the 41 patients hospitalized in this sector were evaluated by the hospital dentistry team, of which $68 \%$ were male and $32 \%$ female. (62\%) in ventilatory support with orotracheal intubation (61\%), with respiratory diseases as the main reason for hospitalization $(46 \%)$. The comparison between the quality of oral health and the incidence of pneumonia associated with mechanical ventilation from August to November did not present significant data that point to a possible relationship among them, probably due to the performance of the hospital dental team integrating the multidisciplinary team active in the unit and the implementation of assistance-related infection control protocols.

Descriptors: Oral Health; Pneumonia, Ventilator-Associated; Intensive Care Units.
\end{abstract}

\section{Resumen}

La cavidad oral es la incubadora ideal para el desarrollo de microorganismos debido a pH, la presencia de nutrientes y factores de retención que facilitan la maduración de la biopelícula, microbios que a menudo está en armonía y equilibrio con el huésped sano, a diferencia de los pacientes hospitalizado en cuidados intensivos. El propósito de este estudio fue evaluar la calidad de la salud oral de los pacientes en la unidad de cuidados intensivos y comparar la incidencia de neumonía asociada a la ventilación mecánica. Se evaluaron todos los registros de admisión en la Unidad de Cuidados Intensivos de Adultos del Hospital Universitario Maria Aparecida Pedrossian HUMAP / Ebserh, del 18 de agosto a 15 de noviembre de, 2016, comparando los datos apropiados con indicadores mensuales Boletines emitidos por el Servicio control de la infección, que muestra las tasas de ventilador infección neumonía asociada correspondiente al mismo período. Se observó que de los 41 pacientes admitidos en ese sector en el período analizado, 37 (90\%) pacientes fueron evaluados por el equipo dental hospital, entre éstos el $68 \%$ eran varones y el $32 \%$ eran de sexo femenino, edad de 30 a 70 años $(62 \%)$ en la intubación traqueal con soporte ventilatorio $(61 \%)$ con enfermedades respiratorias como la causa principal de hospitalización (46\%). La comparación entre la calidad de la salud oral y la incidencia de neumonía asociada a la ventilación mecánica no mostró datos significativos que apuntan a una posible relación entre ellos, probablemente debido a la actuación del personal dental hospital.

Descriptores: Salud Bucal; Neumonía Asociada al Ventilador; Unidades de Cuidados Intensivos.

\section{INTRODUÇÃO}

O comprometimento da cavidade bucal apresenta-se como uma condição desfavorável que interfere no prognóstico de pacientes com complicações sistêmicas, nos quais as infecções bucais podem contribuir para a instalação de condições inflamatórias sistêmicas como a síndrome da resposta inflamatória sistêmica e SEPSE e servir de fonte de 
disseminação de microrganismos por via hematogênica ou através do trato respiratório ${ }^{1}$. Pacientes hospitalizados na maioria dos casos não apresentam higiene bucal satisfatória em virtude do seu quadro sistêmico e, por vezes, da necessidade de intubação orotraqueal, o que proporciona uma abertura bucal constante e gera desidratação da mucosa bucal e queda no fluxo salivar, favorecendo assim o surgimento de comorbidades infeciosas ${ }^{2,3}$.

A doença periodontal é caracterizada por ser uma doença infecciosa e de natureza inflamatória que ocasiona a destruição dos tecidos de suporte do elemento dentário devido a ação direta ou indireta de bactérias. O início da doença periodontal deve-se a presença do biofilme microbiano complexo que coloniza as regiões do sulco gengival entre a superfície dental e as margens gengivais, através de interações de adesão específicas e acúmulo crescente devido a alterações anatômicas, como perda de aderência e formação de bolsa periodontal ${ }^{4,5}$.

A cavidade bucal pode abrigar em torno de 700 espécies bacterianas que participam na formação do biofilme sobre as superfícies dentárias e língua, sofrendo alterações em sua composição tornando-se um reservatório de patógenos respiratórios principalmente em pacientes hospitalizados, o que aumenta ainda mais o risco de pneumonia por aspiração em pessoas susceptíveis ${ }^{6}$.

As pneumonias são classificadas primeiramente em dois tipos: pneumonia adquirida na comunidade $\mathrm{e}$ pneumonia nosocomial, sendo que a primeira está associada à infecção por Streptococcus pneumoniae, e acomete pacientes recém hospitalizados, surgindo nas primeiras horas de internação hospitalar. Por sua vez a pneumonia nosocomial se desenvolve entre 48 e 72 horas após a admissão hospitalar, e há ainda uma subclassificação descrita por alguns autores que classificam em pneumonia associada a ventilação mecânica quando os pacientes estão sob suporte ventilatório através de dispositivos invasivos e diferentemente da primeira classificação em que o patógeno causador não estava presente nem estava incubado no paciente no momento da internação. Os patógenos mais comumente envolvidos são bactérias gram-negativas (Pseudomonas aeruginosa e enterobactérias) e grampositivas Staphylococcus aureus ${ }^{7,8}$.

Pequenas quantidades de saliva podem ser aspiradas devido ao mecanismo fisiológico normal, porém em pacientes internados na Unidade de Terapia Intensiva - UTI que apresentam alterações no nível de consciência, a ocorrência de aspirações e a quantidade torna-se frequente e maior, sendo descrito na literatura a aspiração de patógenos da orofaringe para os pulmões. Esses patógenos podem ser derivados do biofilme dentário dos pacientes internados, que sofre algumas modificações devido ao ambiente, passando a ser colonizado por patógenos respiratórios responsáveis pela Pneumonia Associada à Ventilação Mecânica - PAVM que frequentemente ocorre em ambiente de cuidados intensivos e em pacientes que fazem uso da ventilação mecânica. A PAVM apresenta uma prevalência variável de 6 a 50 casos por 100 admissões em UTI e tem morbidade significativa associada, prolongando o tempo de ventilação mecânica, bem como o tempo de permanência na unidade, com todos os custos associados a esse prolongamento ${ }^{3-5,9}$.

O presente estudo tem como objetivo caracterizar a qualidade da saúde bucal de pacientes internados no Centro de Terapia Intensiva Adulto do Hospital Universitário Maria Aparecida Pedrossian HUMAP/EBSERH, no intuito de se obter a frequência de indivíduos afetados pela doença cárie, doença periodontal, edentulismo e uso de prótese odontológica assim como identificar as alterações estomatológicas presentes, podendo dessa forma relacionar com a incidência de pneumonia associada a ventilação mecânica no mesmo período.

\section{MATERIAL E MÉTODO}

Realizou-se um estudo descritivo, quantitativo e transversal com base em informações descritas em prontuário durante o período de 18 de Agosto a 15 de Novembro de 2016. Após aprovação pelo Comitê de Ética em Pesquisa em Seres Humanos da Universidade Federal de Mato Grosso do Sul e do Comitê de Ética da respectiva unidade hospitalar, e após a assinatura do termo de compromisso para uso de prontuários, foram coletadas informações em prontuários de 41 pacientes internados no Centro de Terapia Intensiva do Hospital Universitário Maria Aparecida Pedrossian HUMAP/EBSERH e comparados com boletins mensais de indicadores emitidos pelo Serviço de Controle de Infecção Hospitalar SCIH do respectivo hospital.

Dos 41 pacientes internados na unidade, foram analisados 37 prontuários que possuíam avaliação odontológica registrada em prontuário de admissão específico da especialidade seguindo os critérios de inclusão e exclusão.

Os critérios de inclusão foram todos os pacientes admitidos na unidade com faixa etária de 14 a 100 anos e de ambos os sexos, e os critérios de exclusão foram pacientes com traumatismo panfacial, quilombolas e indígenas. Coletaram-se dados a respeito de gênero, idade, necessidade de suporte respiratório, local de intubação, doença de base, destruição coronária por cárie, fratura, raiz residual, ausências dentárias, prótese fixa, mobilidade dentária, saburra lingual e cálculos supragengivais.

Os dados coletados em prontuário foram tabulados e sumarizados em planilhas do programa computacional Microsoft Excel (versão 2013 para Windows) e comparados aos dados obtidos pelo serviço de controle de infecção hospitalar quanto a incidência de pneumonia associada à ventilação mecânica no centro de terapia intensiva.

\section{RESULTADOS}

Nesse estudo foram avaliados 41 prontuários, porém apenas 37 possuíam prontuários odontológicos de admissão no CTI adulto do Hospital Universitário Maria Aparecida Pedrossian HUMAP/EBSERH de Campo Grande MS. Destes, $68 \%(n=25)$ do gênero masculino e $32 \%(n=12)$ do gênero feminino. A idade dos pacientes variou de 30 a 70 anos cerca de $62 \%(n=23)$. Dos pacientes avaliados neste estudo, $84 \%(\mathrm{n}=31)$ dos pacientes necessitaram de suporte ventilatório, sendo que desses $61 \% \quad(n=19)$ estavam submetidos a ventilação mecânica por tubo orotraqueal, enquanto que $39 \%(n=12)$ estavam submetidos a ventilação mecânica pela traqueostomia. Quanto ao local de intubação, $46 \%(n=18)$ desses pacientes foram entubados em Unidades de Pronto Atendimento UPA (Tabela 1).

No aspecto geral, os pacientes avaliados apresentavam a cavidade bucal comprometida, observandose a presença de saburra lingual em 54\% ( $n=20)$ nos pacientes avaliados e cálculos supragengivais em $51 \%$ $(\mathrm{n}=19)$. Destes, o maior número de pacientes se concentrou no mês de Setembro. Foi observado edentulismo parcial em $35 \%(n=13)$ dos pacientes, enquanto que a proporção de edentulismo total foi de $27 \%(\mathrm{n}=10)$. A taxa de pacientes que apresentaram edentulismo parcial e total 
simultaneamente foi de $11 \%(\mathrm{n}=4)$. Nos meses de agosto e outubro, em apenas 5\% (n=2). Desses pacientes, apenas 10\% $(n=4)$ ficaram sem avaliação odontológica e, como observado na Tabela 2, concluiu-se que houve um maior comprometimento da integridade bucal no mês de Outubro.

Tabela 1. Pacientes admitidos pela Odontologia Hospitalar no Centro de Terapia Intensiva Adulto do Hospital Universitário Maria Aparecida Pedrossian HUMAP/EBSERH

\begin{tabular}{|c|c|c|c|}
\hline \multicolumn{4}{|c|}{ Pacientes admitidos pela odontologia hospitalar no CTI adulto } \\
\hline & & $\mathbf{N}=$ & $\%$ \\
\hline Admissões no CTI & & 41 & ----- \\
\hline Avaliados pela Odontologia & & 37 & $90 \%$ \\
\hline Gênero & Feminino & 12 & $32 \%$ \\
\hline & Masculino & 25 & $68 \%$ \\
\hline Idade & $14-30$ & 5 & $14 \%$ \\
\hline & $30-70$ & 23 & $62 \%$ \\
\hline & $70-100$ & 9 & $24 \%$ \\
\hline Respiração & $\begin{array}{l}\text { Espontânea } \\
\text { Orotraqueal } \\
\text { Traqueostomia }\end{array}$ & $\begin{array}{c}6 \\
19 \\
12\end{array}$ & $\begin{array}{l}16 \% \\
51 \% \\
32 \%\end{array}$ \\
\hline Local de intubação & $\begin{array}{l}\text { UPA } \\
\text { PAM } \\
\text { Outros }\end{array}$ & $\begin{array}{r}17 \\
9 \\
5\end{array}$ & $\begin{array}{l}46 \% \\
24 \% \\
16 \%\end{array}$ \\
\hline Patologia de Base & $\begin{array}{l}\text { Doenças Infectocontagiosas } \\
\text { Doenças do trato urinário } \\
\text { Doenças respiratórias } \\
\text { Doenças Neurológicas } \\
\text { Cirurgia }\end{array}$ & $\begin{array}{r}5 \\
6 \\
17 \\
4 \\
5\end{array}$ & $\begin{array}{l}14 \% \\
16 \% \\
46 \% \\
11 \% \\
14 \%\end{array}$ \\
\hline
\end{tabular}

Tabela 2. Avaliação odontológica dos pacientes admitidos pela Odontologia Hospitalar no Centro de Terapia Intensiva Adulto do Hospital Universitário Maria Aparecida Pedrossian HUMAP/EBSERH

\begin{tabular}{|c|c|c|c|c|c|}
\hline \multicolumn{6}{|c|}{ Avaliação odontológica } \\
\hline & Agosto & Setembro & Outubro & Novembro & $\%$ \\
\hline $\begin{array}{l}\text { Dentição permanente } \\
\text { completa }\end{array}$ & 3 & 2 & 1 & 1 & $19 \%$ \\
\hline Saburra & 7 & 4 & 7 & 2 & $54 \%$ \\
\hline Cálculo supragengival & 5 & 6 & 6 & 2 & $51 \%$ \\
\hline Edentulismo parcial & 4 & 4 & 4 & 1 & $35 \%$ \\
\hline Edentulismo total & 3 & 2 & 4 & 1 & $27 \%$ \\
\hline $\begin{array}{l}\text { Edentulismo parcial + } \\
\text { Total }\end{array}$ & 1 & 1 & 2 & 0 & $11 \%$ \\
\hline Raiz Residual & 1 & 0 & 1 & 0 & $5 \%$ \\
\hline $\begin{array}{l}\text { Destruição coronária } \\
\text { por cárie }\end{array}$ & 1 & 0 & 1 & 1 & $8 \%$ \\
\hline Mobilidade & 0 & 1 & 1 & 1 & $8 \%$ \\
\hline Fratura & 0 & 0 & 2 & 0 & $5 \%$ \\
\hline Sem avaliação & 2 & 1 & 0 & 1 & $10 \%$ \\
\hline
\end{tabular}

De acordo com boletins mensais emitidos pelo $\mathrm{SCIH}$, o perfil microbiológico de PAVM no período de Agosto à Novembro apresentou uma incidência maior de Pseudomonas spp. e Acinetobacter baumanii e, comparando a relação entre Outubro que foi o mês que apresentou o maior número de pacientes admitidos com cavidade bucal prejudicada com a incidência de PAVM no mesmo período, os dados apontam um decréscimo de PAVM quando comparada aos meses de Agosto e Setembro (Figura 1), não sendo evidenciado uma correlação entre a integridade da cavidade bucal prejudicada que se apresenta em maiores porcentagens em Outubro, dessa forma não apresentando uma correlação significativa entre ambos.

De acordo ainda com o perfil microbiológico de PAVM, que apontam uma prevalência para Pseudomonas spp. e Acinetobacter baumanii resistentes a carbapenemicos, coletado através de culturas de secreção traqueal observouse um dado diferenciado em um dos pacientes hospitalizados diagnosticado com PAVM nesse período de coleta. Neste a cultura de secreção traqueal feita na admissão do respectivo paciente, na qual apresentou apenas o microrganismo Acinetobacter baumanii porém, 6 dias após a primeira coleta, foi realizado nova coleta de sulco gengival, o que evidenciou a presença de Acinetobacter baumanii e Pseudomonas aeruginosas e, 38 dias após, uma terceira coleta foi realizada evidenciando a presença de Pseudomonas aeruginosas em cultura de secreção traqueal.

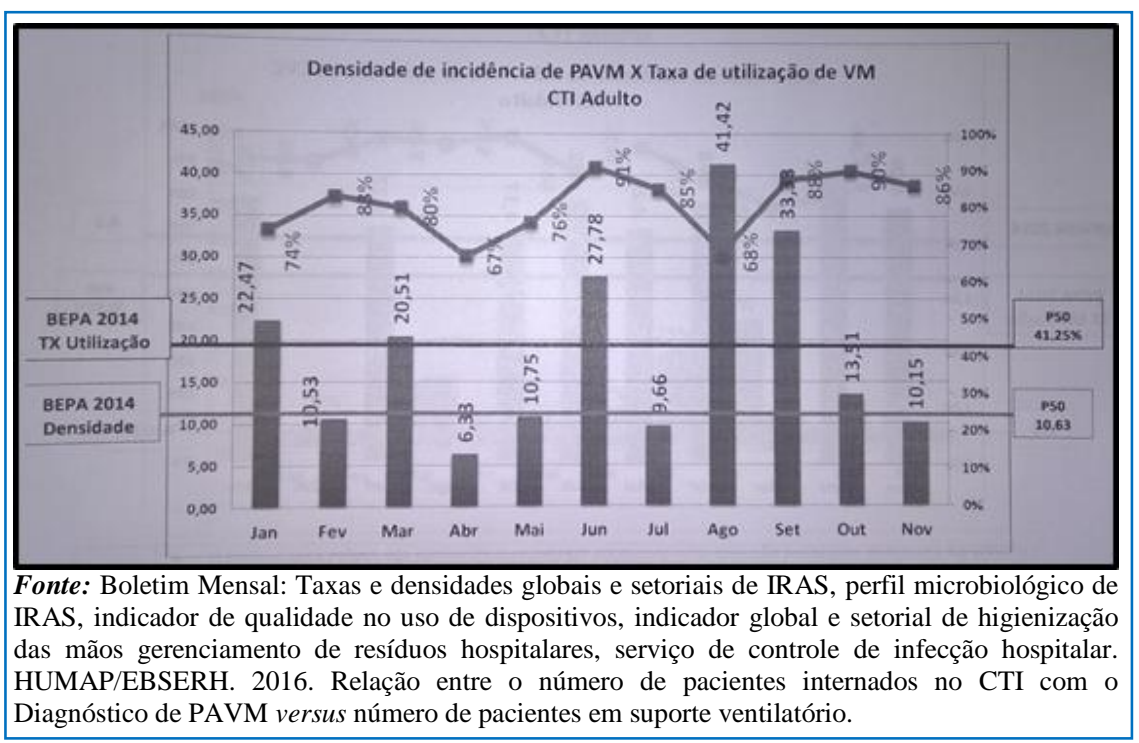

Figura 1: Densidade de incidência de PAVM X Taxa de Utilização de VM - CTI Adulto.

\section{DISCUSSÃO}

De acordo com Baeder et al. ${ }^{10}$, pacientes internados em UTIs apresentam comumente condições orais préexistentes como cáries, doença periodontal e ausência de dentes, com dados que mostram que, desses pacientes, $13 \%$ apresentaram dentes cariados; abscessos em 21\%; doenças gengivais em $21 \%$ e presença de próteses e ferimentos em $46 \%$ dos casos, dados esses que se assemelham aos resultados obtidos no presente estudo. Esses acometimentos prévios somam-se, muitas vezes, a condições como halitose, úlceras traumáticas, saburra lingual e candidíase durante o período de internação, podendo favorecer um maior acometimento da saúde e do bem-estar geral dos pacientes críticos. Situação semelhante foi observada nos achados clínicos dos pacientes do presente estudo, uma vez que a saburra lingual e doença periodontal mostraram-se evidentes, assim como diminuição do fluxo salivar e o ressecamento da mucosa labial.

Neste sentido, o crescimento da microbiota bucal é favorecido pelo ressecamento lábial, higiene bucal deficiente, abertura bucal constante durante os períodos de intubação orotraqueal e pela xerostomia decorrente do uso de inúmeros fármacos ${ }^{10,11}$. Dos pacientes avaliados, a porcentagem dos que apresentaram saburra lingual, cálculo supragengival e edentulismo parcial ou total mostrou-se significativa, o que demonstrou um autocuidado prejudicado. Observou-se o início de uma doença periodontal, resultante de um desequilíbrio no sistema imunológico. Por sua vez a saburra lingual é definida como uma alteração relativamente comum, formada através do acúmulo de restos alimentares, células descamadas, fungos, bactérias e enzimas ativas que participam do processo de digestão $^{4,12}$.

Estruturas da cavidade bucal como dentes, gengivas e língua, representam um reservatório para os patógenos respiratórios, que podem estar presentes durante a internação em UTI, decorrente da substituição da microbiota normal por tais patógenos que, por sua vez, podem colonizar o trato respiratório tornando os pacientes hospitalizados mais suscetíveis a desenvolver uma pneumonia nosocomial. Tal fato é reafirmado por Teng et al. ${ }^{13}$ que sugere que enzimas bacterianas provenientes do biofilme bucal promovem uma 
alteração bioquímica da superfície bucal do paciente, aumentando a adesão e consequentemente sua colonização por patógenos respiratórios ${ }^{14,15}$.

Um estudo realizado por Oliveira et al. ${ }^{16}$ descreve uma amostra de 30 pacientes em que foram encontrados $70 \%$ de patógenos respiratórios no biofilme dental, 63,33\% no dorso da língua e 73,33\% no filtro do respirador artificial, corroborando com a literatura sobre tudo na necessidade de uma maior investigação que aponte o perfil microbiológico da cavidade bucal dos pacientes admitidos no CTI, em decorrência de uma incidência significativa de suporte ventilatório através de intubação orotraqueal e traqueostomia em $84 \%(n=31)$ e de um expressivo número de pacientes com comprometimento na integridade da cavidade bucal.

De acordo ainda com o estudo realizado, dos 30 pacientes avaliados pelo autor, o resultado do aspirado traqueal demonstrou que uma das bactérias mais freqüentemente encontradas em seu estudo foram: Pseudomonas aeruginosa em seis pacientes (20\%) sendo que a bactéria estava presente no filtro do umidificador, em cinco ela estava presente além do filtro do umidificador também no biofilme dental e na língua enquanto que o Acinetobacter calcoaceticus baumanii foram encontrados em um paciente $(3,3 \%)$ nos três locais observados, ou seja, no biofilme dental, biofilme da língua e no tubo umidificador, semelhante ao achado em um dos pacientes com diagnóstico de PAVM do presente estudo no qual foi encontrado no interior do sulco gengival Pseudomonas aeruginosas e Acinetobacter baumanii, patógenos respiratórios frequentemente associados ao quadro clínico de PAVM, salientando a possibilidade de colonização desses patógenos respiratórios em cavidade bucal ${ }^{16}$.

Como descrito na literatura, bactérias presentes no biofilme bucal podem facilitar a colonização das vias aéreas superiores por patógenos pulmonares, ocasionando uma disseminação de patógenos resultantes de uma higiene bucal deficiente para o pulmão em pequenas e grandes quantidades. Isso compromete ainda mais o sistema imunológico dos pacientes internados e demonstra um risco aumentado aos pacientes desse estudo, pois dos avaliados $80 \%$, apresentavam cavidade bucal prejudicada e mesmo assim não foi evidenciado uma maior incidência de PAVM no respectivo período da amostra, em decorrência da influência de diversos fatores como diagnóstico de PAVM impreciso e ação da equipe de odontologia hospitalar no controle de focos infecciosos e da implantação da ação de higiene bucal utilizando a clorexedina a $0,12 \%$ dentro dos protocolos de controle de infecção relacionada a assistência $^{17-19}$.

A American Thoracic Society ${ }^{20}$ define que a PAVM é a infecção nosocomial mais comum em UTIs, com taxas que ficam em torno de 6 a 50 casos por 100 admissões na unidade, segundo Rello et $\mathrm{al}^{21}$. De acordo com Bekaert et $\mathrm{al}^{19} \mathrm{a}$ incidência dessa infecção aumenta com a duração da ventilação mecânica e aponta taxas de ataque de aproximadamente $3 \%$ por dia durante os primeiros 5 dias de ventilação, possuindo uma morbidade significativa associada, prolongando o tempo de ventilação mecânica e consequentemente o tempo de internação hospitalar. Isso é observado na UTI da respectiva unidade hospitalar, onde a rotatividade de pacientes é diminuída quando comparada a de outras unidades hospitalares, possivelmente devido ao perfil de pacientes hospitalizados, pois em grande parte apresentam quadros infecciosos de difícil resolução e com uma faixa etária elevada, muitas vezes apresentando um difícil desmame da ventilação mecânica ${ }^{19,22}$.
O diagnóstico impreciso da PAVM limita a possibilidade de comparação entre diferentes estudos e estratégias de aferição baseadas na utilização das taxas como marcador de qualidade assistencial ${ }^{8,23}$, sendo evidenciado que as taxas registradas pelas equipes de vigilância epidemiológica são significativamente inferiores àquelas assinaladas pelas equipes assistentes nas UTIs há uma certa dificuldade em afirmar corretamente que a PAVM ocorreu necessariamente no CTI da unidade estudada visto que, dos pacientes admitidos no mês de Agosto ao mês de Novembro, $84 \%$ apresentavam-se em suporte ventilatório, sendo que $51 \%$ através de intubação orotraqueal (em grande maioria proveniente das unidades de pronto atendimento) e $32 \%$ eram traqueostomizados ${ }^{24,25}$

Tem se relacionado o uso de solução oral de clorexedina a $0,12 \%$ como uma alternativa na prevenção e no tratamento de doenças bucais possuindo mínimos efeitos colaterais, baixa toxicidade local e sistêmica e não causar alterações da flora local, tornando-se um dos agentes microbianos mais potentes e estudados. Apresenta alta eficácia, sendo classificada como padrão ouro na eliminação do número de microrganismos aeróbios e anaeróbios na saliva (80 a 90\%) demonstrando também um potencial fungicida e com importante substantividade, ou seja, capacidade de permanecer retida no local de ação ativamente, sendo liberada lentamente, evitando a neutralização pelo fluxo salivar ${ }^{25}$.

Rabelo et al. ${ }^{26}$ já descreviam que a presença de placa bacteriana na boca pode influenciar as terapêuticas médicas, devido aos fatores de virulência dos microrganismos que nela se encontram, apresentando maiores probabilidades quando associadas a um comprometimento da integridade bucal como presença de doença periodontal, cáries, necrose pulpar, lesões em mucosa, dentes fraturados ou infectados, traumas provocados por próteses fixas ou movéis. Isso traz repercussões na condição sistêmica de pacientes, principalmente os hospitalizados.

Abidia $^{27}$ em 2007 relatou a necessidade de um Cirurgião Dentista no corpo clínico hospitalar que presta assistência aos pacientes sistemicamente comprometidos, para oferecer um suporte no diagnóstico de alterações orais e como coadjuvante na terapêutica médica, atuando tanto em procedimentos emergenciais como traumas como também em procedimentos preventivos e no agravamento da condição sistêmica ou surgimento de infecções hospitalares como a PAVM, realizando procedimentos curativos e restauradores que visam uma adequação do meio bucal, oferecendo um maior conforto ao paciente durante o processo de internação hospitalar.

Embora a maioria dos pacientes avaliados no presente estudo apresentasse um comprometimento da cavidade bucal e estivesse submetida a suporte ventilatório, a incidência de PAVM na unidade apresentou decréscimo entre os meses de Agosto a Novembro, ao contrário da incidência de comprometimento bucal que foi menor no período. Isso é inversamente proporcional ao mês de Outubro, em que os índices de PAVM apresentavam-se em decréscimo e os índices de comprometimento bucal aumentado, o que possivelmente seja decorrente da atuação da equipe de odontologia hospitalar presente no CTI e da implantação de protocolos de higiene bucal utilizando soluções orais a base de digluconato de clorexedina a $0,12 \%$, resultando em prevenção e estabilização de processos infecciosos ativos presentes na cavidade bucal, assim como controle microbiológico que pudesse favorecer a instalação de patógenos respiratórios. 


\section{CONCLUSÃO}

Diante dos dados expostos no presente estudo, uma relação intrínseca entre a integridade da cavidade bucal e comorbidades respiratórias, tais como a pneumonia associada à ventilação mecânica, existe devido à presença de patógenos respiratórios colonizando o biofilme bucal de pacientes hospitalizados e que apresentam uma cavidade bucal prejudicada previamente. Faz-se necessário um diagnóstico mais preciso de PAVM, juntamente com um estudo microbiológico de cavidade bucal desses pacientes durante a hospitalização.

A presença do Cirurgião Dentista nas equipes assistenciais favorece a prevenção e o controle de comorbidades que possam agravar o quadro clínico geral do paciente e levar a uma hospitalização prolongada e, consequentemente, a um aumento nos custos hospitalares.

\section{REFERÊNCIAS}

1. São Paulo. Secretaria de Saúde. Manual de odontologia hospitalar. São Paulo: Grupo Técnico de Odontologia Hospitalar; 2012.

2. Toledo G, Cruz I. The importance of the oral hygiene in intensive care unit as a way of prevention of nosocomial infection- sistematic literature review. J Special Nurs Care. 2009; 2(1).

3. Jardim Júnior E, Okamoto AC, Meca LB, Silva PP, Bombarda F, Schweitzer CM. Família Enterobacteriaceae, Acinetobacter baumannii e Pseudomonados na microbiota bucal de pacientes mantidos em unidades de terapia intensiva. Arch Health Invest. 2014; 3(4):40-8.

4. Morais TM, Silva A, Avi AL, Souza PH, Knobel E, Camargo LFA. A importância da atuação odontológica em pacientes em Unidade de Terapia Intensiva. Rev bras ter intensiva. 2006; 18(4):412-7.

5. Holt SC, Ebersole JL. Porphyromonas gingivalis, Treponema denticola and Tannerella forsythia: the "red complex", a prototype polybacterial pathogenic consortium in periodontitis. Periodontology 2000. 2005;38:72-122.

6. Socransky SS, Haffajee AD. Periodontal microbial ecology. Periodontology 2000. 2005; 38:135-87.

7. Benatti FG, Montenegro FLB. A intervenção odontológica colaborando na diminuição das afecções respiratórias dos idosos. Rev Eap/Apcd. 2008; 9(2):1-4

8. Tablan OC, Anderson LJ, Besser R, Bridges C, Hajjeh R. Guidelines for preventing health-care-associated pneumonia, 2003: recommendations of CDC and the Healthcare Infection Control Practices Advisory Committee. MMWR Recomm Rep. 2004. 26; 53(RR-3):1-36.

9. Cortelli JR, Cortelli SC. Periodontite crônica e agressiva: prevalência subgengival e frequência de ocorrência de patógenos periodontais. Rev biociênc. 2003; 9(2):91-6.

10. Baeder FM, Cabral GMP, Prokopowitsch I, Araki AT, Duarte DA, Santos MTBR. Condição odontológica em pacientes internados em unidade de terapia intensiva. Pesq Bras Odontoped Clin Integr. 2012; 12(4):517-20.

11. Padovani MCRL, Souza SAB, Sant'Anna GR, Guaré RO. Protocolo de cuidados bucais na Unidade de Tratamento Intensivo (UTI) Neonatal. Rev bras pesqui saúde. $2012 ; 14(1): 71-80$.
12. Santos PSS, Mariano M, Kallas MS, Vilela MCN Impacto da remoção de biofilme lingual em pacientes sob ventilação mecânica. Rev bras ter intensiva. 2013;25(1):44-48.

13. Teng YT, Taylor GW, Scannapieco F, Kinane DF, Curtis M, Beck JD et al. Periodontal health and systemic disorders. J Can Dent Assoc. 2002; 68(3):188-92.

14. Predengarst V, Hallberg IR, Jahnke H, Kleiman C, Hagell P. Oral health, ventilator-associated pneumonia, and intracranial pressure in intubated patients in a neuroscience intensive care unit. Am J Crit Care. 2009; 18(4):368-76.

15. Pedreira MLG, Kusahara DM, de Carvalho WB, Nuñez SC, Peterlini MA. Oral care interventions and oropharyngeal colonization in children receiving mechanical ventilation. Am J Crit Care. 2009; 18(4):319-28.

16. Oliveira LCBS, Carneiro PPM, Fischer RG, Tinoco EMB. A presença de patógenos respiratórios no biofilme bucal de pacientes com pneumonia nosocomial. Rev bras ter intensiva. 2007; 19(4):428-33.

17. Scannapieco FA, Bush RB, Paju S. Associations between periodontal disease and risk for nosocomial bacterial pneumonia and chronic obstructive pulmonary disease. A systematic review. Ann Periodontol, 2003; 8(1):54-69.

18. Rello J, Diaz E. Pneumonia in the intensive care unit. Crit Care Med. 2003; 31(10):2544-51.

19. Bekaert M, Timsit JF, Vansteelandt S, Depuydt P, Vésin A, Garrouste- Orgeas M et al. Outcomerea Study Group. Attributable mortality of ventilator-associated pneumonia: a reappraisal using causal analysis. Am J Respir Crit Care Med. 2011; 184(10):1133-9.

20. American Thoracic Society; Infectious Diseases Society of America. Guidelines for the management of adults with hospital-acquired, ventilator-associated, and healthcare-associated pneumonia. Am J Respir Crit Care Med. 2005; 171(4):388-416.

21. Rello J, Afonso E, Lisboa T, Ricart M, Balsera B, Rovira A et al. A care bundle approach for prevention of ventilator associated pneumonia. Clin Microbiol Infect. 2013; 19(4):363-9

22. Klompas M, Magill S, Robicsek A, Strymish JM, Kleinman K, Evans RS et al. Objective surveillance definitions for ventilator-associated pneumonia. Crit Care Med. 2012; 40(12):3154-61.

23. Almeida RF, Pinho MM, Lima C, Faria I, Santos P, Bordalo C. Associação entre doença periodontal e patologias sistêmicas. Rev Port Clin Geral. 2006; 22(3): 379-90.

24. Eggimann P, Hugonnet S, Sax H, Toveneau S, Chevrolet JC, Pittet D. Ventilator associated pneumonia: caveats for benchmarking. Intensive Care Med. 2003; 29(11):2086-9.

25. Hortense SR, Carvalho ES, Carvalho FS, Silva RPR, Bastos JRM, Bastos RS. Uso da clorexidina como agente preventivo e terapêutico na odontologia. Rev odontol Univ Cid São Paulo. 2010; 22(2):178-84.

26. Rabelo GD, Queiroz CI, Santos PSS. Atendimento odontológico ao paciente em unidade de terapia intensiva. Arq Med Hosp Fac Cienc Med Santa Casa São Paulo. 2010; 55(2):67-70.

27. Abidia RF. Oral care in the intensive care unit: a review. J Contemp Dent Pract. 2007; 8(1):76-82. 


\section{CONFLITO DE INTERESSES}

Os autores declaram não haver conflitos de interesse.

\section{AUTOR PARA CORRESPONDÊNCIA}

Ellen Cristina Gaetti Jardim

ellengaetti@gmail.com

Submetido em 30/05/2017

Aceito em 08/07/2017 